\title{
A Fas pathway to pulmonary fibrosis
}

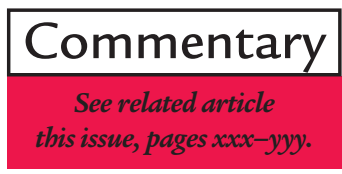

Harold A. Chapman

Department of Medicine, Brigham and Women's Hospital and Harvard Medical School, Boston, Massachusetts 02115, USA

Address correspondence to: Harold A. Chapman, Brigham and Women's Hospital, Thorn Research Building,

Room 803, 20 Shattuck Street, Boston, Massachusetts 02115, USA. Phone: (617) 732-6074; Fax: (617) 232-4623;

E-mail: hchapman@rics.bwh.harvard.edu.

For many years, the inflamed, thickened, and useless alveolar walls of patients with lung fibrosis led investigators to think that the basic process leading to fibrosis was a sustained interstitial pneumonitis. Careful studies of animal models and humans over the last 15 years have revealed that, in fact, most pulmonary fibrosis begins in the alveolus and develops in definable stages over time (Figure 1): (a) epithelial cell injury and alveolar inflammation; (b) organization of the resultant alveolar exudate; and (c) incorporation of the alveolar fibroproliferative process into alveolar walls. Repeating cycles of this series leads to the characteristic signature of distorted and dysfunctional lung parenchyma $(1,2)$. However, an explanation for the earliest recognizable event in this process - loss of alveolar epithelial cells has remained ob-scure. The emergence of the field of programmed cell death (apoptosis) and the realization that lung alveolar epithelial cells, like cells in many tissues, express the Fas death receptor have stimulated new thinking about these early events.

Ligation and clustering of Fas receptors by Fas ligand (expressed on inflammatory cells in Figure 1) results in the assembly of a cytoplasmic protein scaffold containing activated caspase- 8 , the first in a cascade of proteases whose full activation culminates in irreversible cell injury and apoptosis (3). Because apoptotic cells are rapidly cleared by phagocytes, this was thought to be a mechanism of cell death that avoided inflammation. This scenario is best played out in the developing immune system, where fewer than $1 \%$ of $\mathrm{T}$-cell precursors entering the thymus survive selection, many succumbing to a Fasmediated apoptotic death without discernible secondary inflammation (4). However, it is increasingly apparent that under some circumstances ligation of Fas leads to release of proinflammatory cytokines, indicating that, whatever its homeostatic role, Fas ligation can also lead to inflammation and tissue injury $(5,6)$.

In this issue of the JCI, an injurious role for the Fas pathway in the pathogenesis of pulmonary fibrosis is reported (7). In a model of bleomycininduced pulmonary injury and fibrosis, developing 7-14 days after intratracheal injection of bleomycin, mice deficient in either Fas (lpr mutants) or Fas ligand ( gld mutants) were found to be protected from fibrosis (as judged by accumulation of lung hydroxyproline). Epithelial cell apoptosis (as judged by DNA strand breaks) was also attenuated. Similar results were seen when an antagonistic soluble Fas receptor was given intravenously or by inhalation. These observations extend prior work demonstrating that agonist Fas antibodies promote pulmonary fibrosis in mice and that DNA strand breaks consistent with apoptosis are seen in increased numbers of alveolar epithelial cells, both in bleomycin-injured mice and in hu-mans with idiopathic

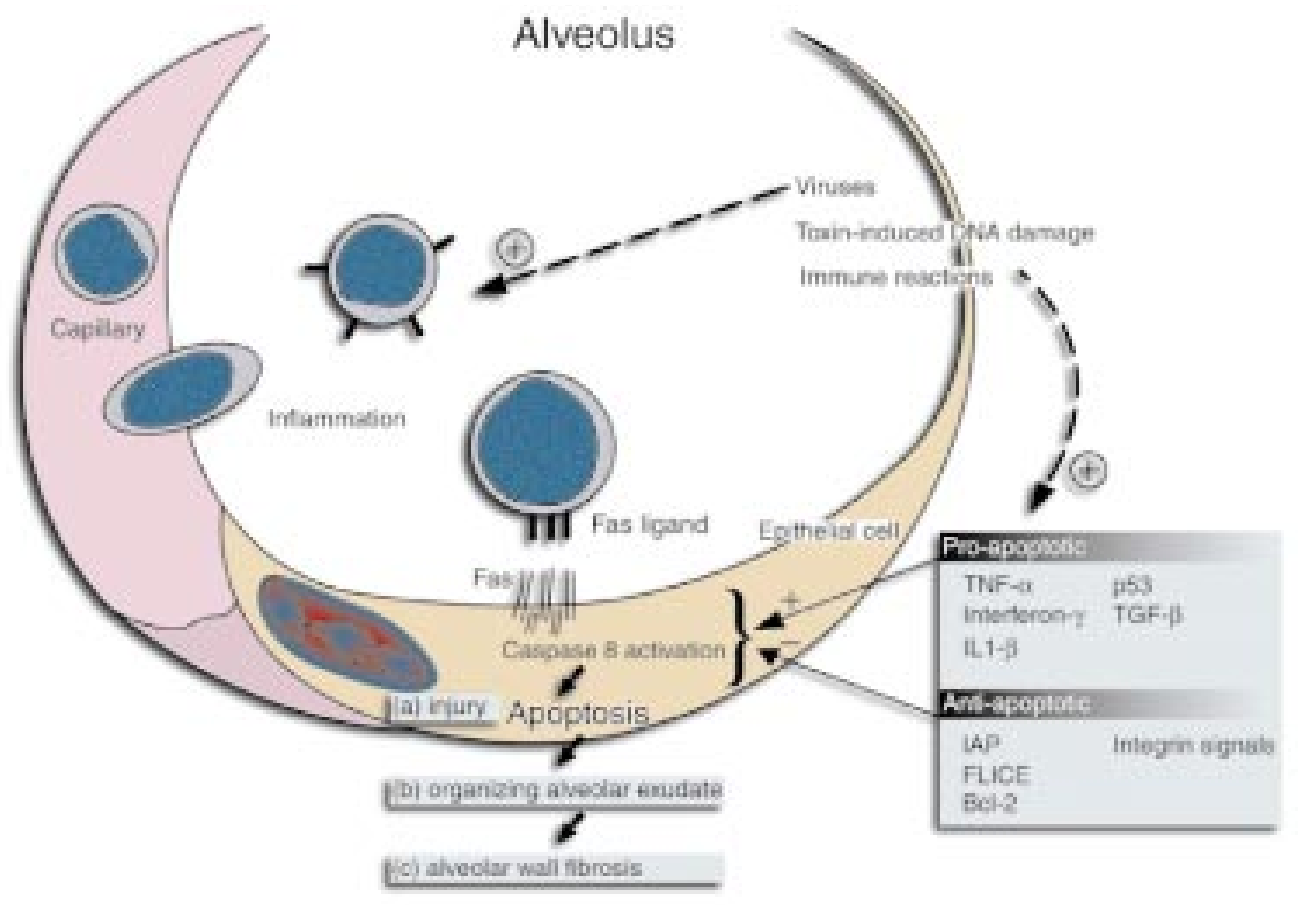

The Journal of Clinical Investigation ｜ July 1999 | Volume 104 | Number 1 
pulmonary fibrosis (IPF) (8-10). These results offer a possible explanation for early loss of epithelial cells seen in pulmonary fibrosis, and imply that this loss is critical to subsequent fibrosis. This seems plausible, because loss of epithelial cell barrier function could be expected to lead to a fibrinous alveolar exudate. An attractive feature of this hypothesis is that initiating agents as disparate as viruses, DNA-damaging toxins (such as bleomycin), and immune reactions are all known triggers of epithelial cell apoptosis. All of these etiologies have been implicated in pulmonary fibrosis in humans.

Although the scenario proposed by Kuwano et al. (7) has attractive features, the picture is far from clear. Possible alterations in lymphocyte cytokine release following loss of Fas/Fas ligand signaling confound the conclusion that epithelial cells are the key beneficiaries of the disrupted Fas pathway. A lymphocytepredominant inflammatory infiltrate is prominent after bleomycin introduction into lungs (7). Furthermore, because of defective T-cell selection, both $l p r$ and $g l d$ mice develop a spontaneous, albeit largely interstitial, lymphocytic pneumonitis. Does altered cytokine release by lymphocytes of the mutant or soluble Fas receptor-treated mice contribute to the protective effect? Fibrosis in the bleomycin model is dependent upon both TNF- $\alpha$ and active TGF- $\beta$, and inflammatory cells and epithelial cells are sources of these cytokines $(11,12)$. This ambiguity could perhaps have been tested by bone marrow transplantation of the Fas mutant mice prior to bleomycin exposure. Second, the Fas pathway is not the only pathway of toxininduced cell death. Lpr mice were not protected from injury in a model of oxygen toxicity (13). This contrasts with a deficiency in plasminogen activator inhibitor-1 (PAI-1), which promotes organization of the fibrinous alveolar exudate (14). PAI1-deficient mice are protected from fibrosis in both bleomycin and oxy- gen models of lung injury $(15,16)$. Thus, consistent with the idea that fibrosis develops as a series of dependent events (Figure 1), alterations at multiple steps, in addition to improved survival of alveolar epithelial cells, could have influenced the final outcome. And lastly, of course, apoptosis is not all bad. In addition to being a mechanism to limit inflammation and possibly effect normal epithelial cell turnover, stimulation of fibroblast apoptosis has been suggested as a mechanism to minimize alveolar wall fibrosis (2).

Despite these limitations, the proposition that Fas-dependent epithelial cell injury and death is an important pathway to pulmonary fibrosis warrants further study. Particularly interesting is emerging evidence of the complicated manner in which cells respond to Fas ligation. Rather than simply triggering apoptosis, the outcome of Fas ligation on the activity of effector caspases depends on the net influence of both proapoptotic and antiapoptotic factors, some of which are listed in Figure 1. Cytokines implicated in lung fibrosis in mice (TNF- $\alpha$, TGF$\beta)$ promote expression of cofactors such as bax and p53, which sensitize cells to Fas receptor clustering and caspase-8 activation (17). Conversely, expression of bcl-2 and similar antiapoptotic factors, as promoted by signaling through integrins and other receptors, provide resistance to Fas-mediated injury. For example, corticosteroids induce a protease inhibitor of effector caspases (IAP) and block Fas-mediated lung epithelial cell death in vitro (18). One could envision that genetic or environmentally induced dysregulation of the balance among proapoptotic and antiapoptotic factors on alveolar surfaces could underlie individual susceptibility to IPF and, conceivably, resistance to therapy. Whether or not this is true, understanding and manipulating the regulators of lung epithelial cell death, should provide new insight into the beginnings of this otherwise relentless process.

\section{Acknowledgments}

The author thanks Ed Ingenito, Richard Riese, and Charlotte McKee for helpful comments regarding the manuscript.

1. Kuhn, C., et al. 1989. An immunohistochemical study of architectural remodeling and connective tissue synthesis in pulmonary fibrosis. Am. Rev. Respir. Dis . 140:1693-1703.

2. Bitterman, P.D., and Wendt, C.H. 1998. The pathogenesis of pulmonary fibrosis. In Fishman's pulmonary diseases and disorders. A.P. Fishman, editor. McGraw-Hill. New York, NY. 347-358.

3. Muzio M., Stockwell, B.R., Stennicke, H.R., Salvesen, G.S., and Dixit, V.M. 1997. An induced proximity model for caspase-8 activation. J. Biol. Chem . 272:17907-17911.

4. Ogasawara, J., Suda, T., and Nagata S. 1995 Selective apoptosis of CD4+CD8+ thymocytes by the anti-Fas antibody. J. Exp. Med. 181:485-491.

5. Miwa, K., et al. 1998. Caspase 1-independent IL1 beta release and inflammation induced by the apoptosis induced Fas ligand. Nat. Med. 4:1287-1292.

6. Chen, J.J., Sun, Y., and Nabel, G.J. 1998. Regulation of the proinflammatory effects of Fas ligand (CD95L). Science. 282:1714-1717.

7. Kuwano, K., et al. 1999. Essential roles of the Fas-Fas ligand pathway in the development of pulmonary fibrosis. J. Clin. Invest. 104:13-19

8. Hagimoto, N., et al. 1997. Induction of apoptosis and pulmonary fibrosis in mice in response to ligation of Fas antigen. Am. J. Respir. Cell Mol. Biol. 17:272-278.

9. Hagimoto, N., Kuwano, K., Nomoto, Y., Kunitake, R., and Hara, N. 1997. Apoptosis and expression of Fas/Fas ligand mRNA in bleomycin-induced pulmonary fibrosis in mice. Am. J. Respir. Cell Mol. Biol. 16:91-101.

10. Kunitake, R., et al. 1998. Expression of p53, p21(Waf1/Cip1/Sdi1) and Fas antigen in collagen vascular and granulomatous lung diseases. Eur. Respir. J. 12:920-925.

11. Piguet, P.F., and Vesin, C. 1994. Treatment by human recombinant soluble TNF receptor of pulmonary fibrosis induced by bleomycin or sili$\mathrm{ca}$ in mice. Eur. Respir. J. 7:515-518.

12. Munger, J.S., et al. 1999. The integrin $\alpha v \beta 6$ binds and activates latent TGF $\beta 1$ : a mechanism for regulating pulmonary inflammation and fibrosis. Cell. 96:319-328.

13. Barazzone, C., Horowitz, S., Donati, Y.R. Rodriguez, I., and Piguet, P.F. 1998. Oxygen toxicity in mouse lung: pathways to cell death. Am. J. Respir. Cell Mol. Biol. 19:573-581.

14. Chapman, H.A. 1997. Plasminogen activators, integrins, and the coordinant regulation of cell adhesion and migration. Curr. Opin. Cell Biol. 9:714-724.

15. Barazzone, C., Belin, D., Piguet, P.F., Vassalli, J.D., and Sappino, A.-P. 1996. Plasminogen activator inhibitor-1 in acute hyperoxic mouse lung injury. J. Clin. Invest . 98:2666-2673.

16. Eitzman, D.T., et al. 1996. Bleomycin-induced pulmonary fibrosis in transgenic mice that either lack or overexpress the murine plasminogen activator inhibitor-1 gene. J. Clin. Invest. 97:232-237.

17. Tschopp, J., Irmler, M., and Thome, M. 1998 Inhibition of Fas death signals by FLIPs. Curr. Opin. Immunol . 10:552-558.

18. Wen, L.P., Madani, K., Fahrni, J.A., Duncan, S.R., and Rosen, G.D. 1997. Dexamethasone inhibits lung epithelial cell apoptosis induced by IFN- $\gamma$ and Fas. Am. J. Physiol. 273:L921-L929. 